\title{
A prospective cohort study of Staphylococcus aureus and methicillin-resistant Staphylococcus aureus carriage in neonates: the role of maternal carriage and phenotypic and molecular characteristics
}

This article was published in the following Dove Press journal:

Infection and Drug Resistance

Jialing Lin'

Chuanan $\mathrm{Wu}^{2}$

Chunrong Yan²

Qianting $\mathrm{Ou}^{\prime}$

Dongxin Lin'

Junli Zhou'

Xiaohua Ye'

Zhenjiang Yao'

'Department of Epidemiology and Health Statistics, School of Public Health, Guangdong Pharmaceutical University, Guangzhou, China; ${ }^{2}$ Maternal and Child Health Care Center of Longhua District, Shenzhen, China

Correspondence: Zhenjiang Yao Department of Epidemiology and Health Statistics, School of Public Health, Guangdong Pharmaceutical University, 283 Jianghai Street, Guangzhou 510310 China

Tel +86203405 5806

Fax +86 2034055355

Email zhjyao2001@yahoo.com
Background: Staphylococcus aureus, particularly methicillin-resistant S. aureus (MRSA), in mothers can cause serious outcomes in neonates. We aimed to elucidate the associations of S. aureus and MRSA carriage between mothers and neonates.

Methods: A prospective cohort study was conducted between August and November 2015 in two hospitals in Shenzhen, China. Chinese pregnant women and their neonates who met the inclusion criteria were included in this study; samples and relevant information were collected. We assessed maternal-neonatal associations by using Poisson regression models.

Results: Overall, 1834 mothers and their neonates were included in this study. The prevalence of isolate carriage among the mothers was as follows: S. aureus (nasal, 25.8\%; vaginal, 7.3\%; and nasal and vaginal, 3.3\%) and MRSA (nasal, 5.7\%; vaginal, 1.7\%; and nasal and vaginal, $0.5 \%$ ). The incidences of $S$. aureus and MRSA carriage among neonates were $3.3 \%$ and $0.8 \%$, respectively. Of the 21 maternal-neonatal pairs with $S$. aureus carriage, 14 were concordant pairs with the same phenotypic and molecular characteristics. After adjustment, the relative risks and $95 \%$ confidence intervals (CIs) between the $S$. aureus carriage of neonates and nasal S. aureus carriage, vaginal $S$. aureus carriage, and both nasal and vaginal $S$. aureus carriage of mothers were 2.8 (95\% CI, 1.6-4.8), 7.1 (95\% CI, 4.1-12.4), and 9.6 (95\% CI, 4.2-22.4), respectively. Conclusion: $S$. aureus carriage in mothers increases the risk for neonates.

Keywords: Staphylococcus aureus, methicillin-resistant Staphylococcus aureus, neonates, mothers, maternal-neonatal association

\section{Introduction}

Staphylococcus aureus and methicillin-resistant $S$. aureus (MRSA) infections have been recently increasing in pregnant women and neonates hospitalized in neonatal intensive care units (NICUs). ${ }^{1,2}$ Much of this increase has been reported to be driven by the rise of community-associated MRSA (CA-MRSA), which commonly causes infections in patients without traditional influencing factors. ${ }^{1,3}$

$S$. aureus and MRSA have been reported to commonly colonize the vagina of women aged 11-60 years, particularly in those aged $31-40$ years. ${ }^{4}$ Pregnant women are more likely to have vaginal carriage of $S$. aureus and MRSA than non-pregnant women because of their increased estrogen and blood glucose levels. ${ }^{5}$ The prevalence of $S$. aureus and MRSA carriage in pregnant women has been reported to range 
from $13 \%$ to $22 \%$ and from $0.5 \%$ to $10 \%$, respectively. ${ }^{6-13}$ Regarding the neonate population, CA-MRSA has become the most frequent type of $S$. aureus, causing diseases in the NICU and even among healthy full-term neonates. ${ }^{1,14-16}$ It has been reported that maternal carriage of $S$. aureus and MRSA plays a role in neonatal carriage. ${ }^{13,17-23}$ The neonates' increased vulnerability to $S$. aureus and MRSA carriages provides a strong impetus to limit the acquisition and spread of $S$. aureus and MRSA in this population. We require the complete understanding of the modes of transmission of $S$. aureus and MRSA to neonates. However, those previous studies lacked adequate sample sizes or failed to analyze the phenotypic and molecular features of $S$. aureus and MRSA isolates between mothers and neonates.

Although maternal-neonatal transmission is significant for several infectious agents, such as group B streptococcus and gram-negative enteric bacilli, ${ }^{24,25}$ similar published data for $S$. aureus and MRSA in China are lacking. Furthermore, vaginal carriage of $S$. aureus and MRSA at delivery and vertical transmission of $S$. aureus and MRSA from mothers to their neonates have also never been systematically studied in China.

Therefore, we designed a prospective cohort study to determine the status of $S$. aureus and MRSA carriage of mothers and their neonates at delivery. The goals of the current study were 1) to determine the prevalence of $S$. aureus and MRSA carriage in pregnant women at two hospitals in Shenzhen, China, 2) to define the incidence of $S$. aureus and MRSA carriage in neonates delivered by enrolled pregnant women, 3) to determine the associations between mothers and neonates with $S$. aureus and MRSA carriage based on culture results and phenotypic and molecular characteristics, and 4) to estimate the influencing factors of $S$. aureus and MRSA carriage in neonates through a questionnaire given to the mothers and a medical records review.

\section{Patients and methods}

\section{Ethics statement}

This study was approved by the ethics committee of Guangdong Pharmaceutical University, and it was performed in accordance with the approved guidelines. All participants signed an informed consent form.

\section{Study design and subjects}

A prospective cohort study was conducted between August and November 2015 in two hospitals in Shenzhen, China. The two hospitals, Longhua Central Hospital and Guanlan People's Hospital, are large hospitals whose obstetric service delivers between 10000 and 15000 neonates per year; these are two of the largest delivery hospitals in China. All included pregnant women were inpatients because of parturition. The inclusion criteria were as follows: 1) pregnant Chinese women, 2) between 35 and 40 weeks of gestation, 3) provision of signed informed consent, 4) absence of acute diseases, and 5) no receipt of a vaginal disinfection examination. Those who did not meet the criteria were excluded.

\section{Data collection and sampling}

A face-to-face questionnaire was administered by trained personnel that aimed to collect demographic information and information regarding potential factors influencing $S$. aureus and MRSA carriage during pregnancy. Medical records of the pregnant women and their neonates were reviewed by two members of the study team who were blinded to the maternal and neonatal $S$. aureus and MRSA carriage status.

Sterile swabs moistened with sterile saline water were used to sample both the nares and vagina of the enrolled pregnant women prior to delivery by trained personnel. Each pregnant woman provided two samples from two sites: a nasal sample and a vaginal sample. As soon as possible after delivery, when the neonate had been towel dried, a swab was obtained from the neonate's nares, oral cavity, and skin (axilla, area around the eyes, periumbilical area, and groin with the same swab) by the trained nursing staff within 2 hours of delivery. There are two swabs from each mother and one swab from each neonate. All swabs were then inoculated into enrichment broth tubes containing 1\% tryptone, $7.5 \%$ sodium chloride, $1 \%$ mannitol, and $0.25 \%$ yeast extract.

\section{Isolation and identification of S. aureus and MRSA}

After 24 hours of incubation at $37 \pm 1^{\circ} \mathrm{C}$, the swabs were transferred to mannitol salt agar plates for another 24 hours of incubation. The samples were identified as $S$. aureus isolates when they had specific colony morphology and were positive for gram staining, catalase reaction, hemolysis test, DNase test, and coagulase tests. Those $S$. aureus isolates that were resistant to cefoxitin were identified as MRSA isolates. We isolated one isolate per sample.

\section{Antibiotic susceptibility testing}

Antibiotic resistance testing was conducted by using the Kirby-Bauer disk diffusion method, following the guidelines of the Clinical and Laboratory Standards Institute of 2015. All $S$. aureus isolates underwent phenotypic analysis for antibiotic resistance to 11 antimicrobial agents: cefoxitin, clindamycin, rifampicin, moxifloxacin, tobramycin, 
gentamicin, sulfamethoxazole-trimethoprim, linezolid, teicoplanin, and erythromycin. Isolates were defined as resistant if they were resistant or intermediate to the antibiotic. They were classified as multidrug resistant (MDR) if they were resistant to no less than three antibiotic classes. ${ }^{26}$ The S. aureus isolate ATCC25923 (American Type Culture Collection, Manassas, VA, USA) was used for quality control.

\section{Molecular characteristics}

The DNA of all $S$. aureus isolates was extracted by using the sodium dodecyl sulfate method. ${ }^{27}$ All $S$. aureus isolates were further tested to confirm the presence of the toxin genes including Panton-Valentine leukocidin $(P v l)$, toxic shock syndrome toxin (Tst), exfoliative toxin A (Eta), and exfoliative toxin $\mathrm{B}(E t b)$ by using polymerase chain reaction (PCR) assays as in previous studies. ${ }^{28,29}$

A multiplex PCR technique was used to confirm the type of staphylococcal cassette chromosome mec (SCC$m e c)$ gene. ${ }^{30}$ The results were reported as types $\mathrm{I}-\mathrm{V}$, and those isolates that were not types $\mathrm{I}-\mathrm{V}$ were deemed as non-typeable (NT). SCCmec types I-III were classified as hospital-associated MRSA (HA-MRSA), whereas types IV and $\mathrm{V}$ were classified as CA-MRSA.

The multilocus sequence typing (MLST) PCR assays were performed by using previously published primers and conditions. ${ }^{31}$ Allelic profiles and sequence types (STs) were assigned by using the MLST database (http://www.mlst. net). Singletons or members of a clonal complex (CC) were determined by using the eBURST algorithm (http://eburst. mlst.net). A dendrogram analysis was performed based on ST types to determine the clonal relatedness and potential epidemiologic origin.

\section{Statistical analysis}

The significant difference between proportions was determined by using Pearson's chi-square tests or Fisher's exact tests for categorical variables. To identify variables that might confound the association of $S$. aureus and MRSA carriage between mothers and neonates, we assessed the difference of influencing factors among mothers and neonates with a univariate analysis (Pearson's chi-square tests or Fisher's exact tests). Influencing factors independently associated with $S$. aureus and MRSA carriage among neonates were identified as adjustment variables using the Poisson regression model to estimate the correlations of isolates between mothers and neonates. Relative risks (RRs) and 95\% confidence intervals (CIs) were used to assess the maternal-neonatal relatedness of $S$. aureus and MRSA carriage. In addition, we used adjusted RRs (aRRs) and the carriage probability of the control group to calculate the number needed to harm (NNH) for each outcome according to a published formula. ${ }^{32}$ A two-sided $P$-value for statistical significance was defined as $P<0.05$. The sample size was calculated by using power two proportions method with $4.00 \%$ proportion, 4.00 ratio, 0.05 alpha, and 0.80 power. Taking $10 \%$ loss of follow-up into consideration, the estimated minimum sample size of this study was 107 for each group. All analyses were performed by using STATA 14.2 (StataCorp LP, College Station, TX, USA).

\section{Results \\ Prevalence of S. aureus and MRSA carriage among mothers}

A total of 2172 pregnant women consented to participate in this study. However, 338 pregnant women were excluded because of lack of medical records of their neonates. We then completed enrollment and microbiological, phenotypic, and molecular analyses of 1834 pregnant women and their neonates within 4 months. The prevalence of $S$. aureus and MRSA nasal carriage among pregnant women was $25.8 \%$ (473/1834) and 5.7\% (104/1834), respectively. The prevalence of $S$. aureus and MRSA vaginal carriage among pregnant women was $7.3 \%(133 / 1834)$ and $1.7 \%(31 / 1,834)$, respectively. The prevalence of $S$. aureus and MRSA with both nasal and vaginal carriage among pregnant women was $3.3 \%(60 / 1834)$ and $0.5 \%(9 / 1834)$, respectively. There were no statistically significant differences of $S$. aureus (nasal: $\chi^{2}=0.985, P=0.321$; vaginal: $\chi^{2}=0.328, P=0.567$; and nasal and vaginal: $\chi^{2}=2.413, P=0.299$ ) and MRSA (nasal: $\chi^{2}=0.252$, $P=0.616$; vaginal: $\chi^{2}=0.075, P=0.784$; and nasal and vaginal: $\chi^{2}=1.858, P=0.553$ ) carriage among pregnant women in the two different hospitals.

\section{Incidence of S. aureus and MRSA carriage among neonates}

The incidences of $S$. aureus and MRSA carriage in the neonates were $3.3 \%(60 / 1834)$ and $0.8 \%(15 / 1834)$, respectively. In the $133 \mathrm{~S}$. aureus vaginal carriage pregnant women, the incidences of $S$. aureus and MRSA carriage in their neonates were $15.8 \%(21 / 133)$ and $2.3 \%(3 / 133)$, respectively. In the 1701 non-S. aureus vaginal carriage pregnant women, the incidences of $S$. aureus and MRSA carriage in their neonates were $2.3 \%(39 / 1701)$ and $0.7 \%(12 / 1701)$, respectively. There were no statistically significant differences of $S$. aureus $\left(\chi^{2}=0.494, P=0.482\right)$ and MRSA $\left(\chi^{2}=1.598\right.$, $P=0.206$ ) carriage among the neonates in the two different hospitals. 


\section{Maternal-neonatal association for}

\section{S. aureus and MRSA carriage}

There was a significant difference between $S$. aureus carriage of neonates and $S$. aureus carriage of mothers $\left(\chi_{\text {trend }}^{2}=4.418\right.$, $P=0.036$ ). The RRs and $95 \%$ CIs between the $S$. aureus carriage of neonates and S. aureus nasal carriage, S. aureus vaginal carriage, and $S$. aureus nasal and vaginal carriage of mothers were 2.7 (95\% CI, 1.6-4.4), 6.9 (95\% CI, 4.1-11.7), and 10.2 (95\% CI, 4.6-22.5), respectively. There was no significant difference between MRSA carriage of neonates and the number of MRSA isolates of mothers $\chi_{\text {trend }}^{2}=0.230$, $P=0.631$ ). The RRs and 95\% CIs between MRSA carriage of the neonates and MRSA nasal carriage and MRSA vaginal carriage of the mothers were 2.6 (95\% CI, 10.6-11.3) and 4.2 (95\% CI, 0.6-31.6), respectively. There was no statistically significant association of MRSA carriage between mothers and neonates (Table 1).

In the multivariate analysis, vaginal delivery (RR, 2.9; 95\% CI, 1.2-6.7), low frequency of vaginal examination after hospitalization $(\leq 2)(\mathrm{RR}, 1.8 ; 95 \% \mathrm{CI}, 1.0-3.0)$, and male gender of neonates (RR, 1.7; 95\% CI, 1.0-3.0) were risk factors for $S$. aureus carriage among neonates, whereas premature rupture of membranes (RR, $0.5 ; 95 \%$ CI, 0.2-1.7) was no longer statistically associated with $S$. aureus carriage among neonates. Regarding MRSA carriage among neonates, a low frequency of vaginal examination after hospitalization $(\leq 2)(\mathrm{RR}, 5.5$; 95\% CI, 1.5-19.9) was a risk factor. The aRRs and $95 \%$ CIs between the $S$. aureus carriage of neonates and $S$. aureus nasal carriage, $S$. aureus vaginal carriage, and $S$. aureus nasal and vaginal carriage of mothers were 2.8 (95\% CI, 1.6-4.8), 7.1 (95\% CI, 4.1-12.4), and 9.6 (95\% CI, 4.2-22.4), respectively. However, after adjustment, there was still no statistically significant association of MRSA carriage between mothers and neonates (Tables 1 and 2).

In $S$. aureus isolates, the $\mathrm{NNH}$ for nasal carriage of mothers in neonates was 25 (15-71), that for vaginal carriage of mothers was 4 (2-36), and that for both nasal and vaginal carriage of mothers was 33 (28-38). In MRSA isolates, the $\mathrm{NNH}$ for nasal carriage of mothers in neonates was $138(-54$ to 30 ), that for vaginal carriage of mothers was 23 ( -21 to $7)$, and that for both nasal and vaginal carriage of mothers was 42 ( -39 to 12$)$.

In addition, 133 mothers were found to have $S$. aureus vaginal carriage and 60 neonates were found to have $S$. aureus carriage; however, only 21 maternal-neonatal pairs had $S$. aureus carriage. Fourteen of those pairs were concordant pairs for $S$. aureus carriage. Six of these 21 pairs had MRSA carriage, and six were concordant pairs for MRSA carriage (Figure 1).

\section{Phenotypic characteristics of S. aureus and MRSA isolates}

There were no significant differences in antibiotic resistance between $S$. aureus of the mothers and $S$. aureus of the neonates except for teicoplanin. For MRSA isolates, there was no significant difference in antibiotic resistance between nasal MRSA of the mothers and MRSA of the neonates. Regarding the significant difference in antibiotic resistance between vaginal MRSA of the mothers and MRSA of the neonates, only teicoplanin was significant (Table 3). In addition, 14 pairs showed the same antibiotic resistance pattern with the most predominant pattern being resistant to both penicillin and clindamycin $(12,85.7 \%)$ (Figure 1).

\section{Molecular characteristics of S. aureus and MRSA isolates}

There was no significant difference in toxin genes in $S$. aureus between the mothers and $S$. aureus of the neonates except for the Eta gene. There was no significant difference in toxin genes in MRSA isolates between the mothers and neonates (Table 3).

For the SCCmec-type results, the most predominant type among 15 neonatal MRSA isolates was NT (8, 53.3\%),

Table I Multivariate analysis of influencing factors among neonates with Staphylococcus aureus and MRSA carriage in Shenzhen, 20 I5

\begin{tabular}{|c|c|c|c|c|c|c|c|c|}
\hline \multirow{2}{*}{$\begin{array}{l}\text { Microbial carriage } \\
\text { of mothers }\end{array}$} & \multicolumn{4}{|l|}{ S. aureus } & \multicolumn{4}{|l|}{ MRSA } \\
\hline & RR (95\% Cl) & $\operatorname{aRR}(95 \% \mathrm{Cl})^{a}$ & $P$-value ${ }^{a}$ & $\begin{array}{l}\text { NNH } \\
(95 \% \mathrm{Cl})^{\mathrm{a}}\end{array}$ & RR (95\% Cl) & $\operatorname{aRR}(95 \% \mathrm{Cl})^{b}$ & $P$-value ${ }^{b}$ & $\begin{array}{l}\text { NNH } \\
(95 \% \mathrm{Cl})^{b}\end{array}$ \\
\hline Nasal carriage & $2.7(1.6-4.4)$ & $2.8(1.6-4.8)$ & $<0.001$ & $25(|5-7|)$ & $1.7(0.4-7.5)$ & $2.0(0.4-8.9)$ & 0.066 & $138(-54$ to 30$)$ \\
\hline Vaginal carriage & $6.9(4.1-11.7)$ & $7.1(4.1-12.4)$ & $<0.001$ & $6(4-I I)$ & $2.6(0.6-11.3)$ & $2.9(0.6-12.9)$ & 0.067 & $23(-2 \mid$ to 7$)$ \\
\hline $\begin{array}{l}\text { Both nasal and vaginal } \\
\text { carriage }\end{array}$ & $10.2(4.6-22.5)$ & $9.6(4.2-22.4)$ & $<0.001$ & $8(4-46)$ & $4.2(0.6-31.6)$ & $5.5(0.7-41.9)$ & 0.056 & $42(-39$ to 12$)$ \\
\hline
\end{tabular}

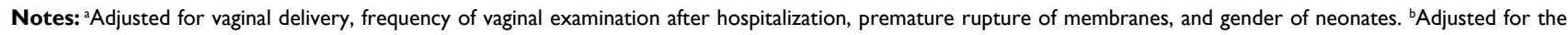
frequency of vaginal examination after hospitalization.

Abbreviations: MRSA, methicillin-resistant S. aureus; RR, relative risk; aRR, adjusted relative risk; $\mathrm{Cl}$, confidence interval; $\mathrm{NNH}$, number needed to harm. 
Table 2 Univariate analysis of influencing factors among neonates with Staphylococcus aureus and MRSA carriage in Shenzhen, 2015

\begin{tabular}{|c|c|c|c|c|c|c|c|}
\hline \multirow[t]{2}{*}{ Influencing factors } & \multicolumn{4}{|c|}{ S. aureus $(n=60)$} & \multicolumn{3}{|l|}{ MRSA $(n=15)$} \\
\hline & $\mathbf{N}$ & Positive (\%) & $\chi^{2}$ & $P$-value & Positive (\%) & $\chi^{2}$ & $P$-value \\
\hline \multicolumn{8}{|l|}{ Mothers } \\
\hline Nasal carriage & 473 & $29(6.1)$ & 16.24 & 0.00 & $2(1.9)$ & NA & 0.21 \\
\hline Vaginal carriage & 133 & $21(15.8)$ & NA & 0.00 & $\mathrm{I}(3.2)$ & NA & 0.23 \\
\hline Both nasal and vaginal carriage & 60 & $9(15.0)$ & NA & 0.00 & $0(0)$ & NA & 0.78 \\
\hline Age $(>35$ years $)$ & 114 & $5(4.4)$ & NA & 0.49 & $0(0)$ & NA & 0.31 \\
\hline Education (below high school) & 1287 & $46(3.6)$ & 1.57 & 0.21 & $12(0.9)$ & NA & 0.38 \\
\hline Vaginal delivery & 1420 & $54(3.8)$ & NA & 0.02 & $13(0.8)$ & NA & 0.54 \\
\hline Natural impregnation & 1809 & $60(3.3)$ & NA & 1.00 & $15(0.8)$ & NA & 1.00 \\
\hline First pregnancy & 606 & $20(3.3)$ & 0.002 & 0.964 & $4(0.7)$ & 0.28 & 0.60 \\
\hline First parturition & 924 & $26(2.8)$ & 1.24 & 0.27 & $6(0.7)$ & 0.66 & 0.42 \\
\hline History of abortion & 660 & $18(2.7)$ & 0.71 & 0.40 & $5(0.8)$ & 0.06 & 0.81 \\
\hline $\begin{array}{l}\text { Frequency of vaginal examination after } \\
\text { hospitalization }(\leq 2)\end{array}$ & 533 & $29(5.4)$ & 5.97 & 0.02 & $10(1.9)$ & NA & 0.007 \\
\hline Vaginitis & 150 & $4(2.7)$ & NA & 0.81 & $\mathrm{I}(0.7)$ & NA & 1.00 \\
\hline Premature rupture of membranes & 364 & $6(1.7)$ & 3.88 & 0.049 & $0(0)$ & NA & 0.05 \\
\hline Days of hospitalization $(>3)$ & 1372 & $40(2.9)$ & 1.53 & 0.22 & $8(0.6)$ & NA & 0.12 \\
\hline Weeks of pregnancy $(<37)$ & 15 & I (6.7) & NA & 0.44 & $0(0)$ & NA & 1.00 \\
\hline Tobacco use during pregnancy & 5 & $0(0)$ & NA & 1.00 & $0(0)$ & NA & 1.00 \\
\hline Antibiotic use during pregnancy & 115 & $3(2.6)$ & NA & 0.68 & $\mathrm{I}(0.9)$ & NA & 1.00 \\
\hline Pet owner & 72 & $3(4.2)$ & NA & 0.51 & I (I.4) & NA & 0.45 \\
\hline \multicolumn{8}{|l|}{ Neonates } \\
\hline Male gender & 991 & $40(4.0)$ & 3.99 & 0.046 & $8(0.8)$ & 0.003 & 0.96 \\
\hline Birth weight $(<2500 \mathrm{~g})$ & 52 & I (I.9) & NA & 1.00 & $0(0)$ & NA & 1.00 \\
\hline Admission to neonatology ward & 162 & $4(2.5)$ & NA & 1.00 & $0(0)$ & NA & 1.00 \\
\hline Apgar Ist minute $\leq 3$ & 0 & $0(0)$ & NA & NA & $0(0)$ & NA & NA \\
\hline Apgar 5 th minute $\leq 6$ & 0 & $0(0)$ & NA & NA & $0(0)$ & NA & NA \\
\hline
\end{tabular}

Notes: $\mathrm{N}$ indicates the number of patients for whom information was available; $\mathrm{n}$ indicates the number of isolates. This was a univariate analysis of influencing factors.

Abbreviations: NA, not applicable; MRSA, methicillin-resistant S. aureus.

followed by type IV (4, 26.7\%), II (2, 13.3\%), and I (1, $6.7 \%)$. The most predominant type among 104 maternal nasal MRSA isolates was type IV $(54,51.9 \%)$, followed by NT (30, 28.9\%), II (12, 11.5\%), and I (8, 7.7\%). The most predominant type among 31 maternal vaginal MRSA isolates was type I $(11,35.5 \%)$, followed by IV $(9,29.0 \%)$, NT ( 8 , $25.8 \%)$, and II $(3,9.7 \%)$. MRSA isolates of both mothers and neonates were from communities.

There were $13 \mathrm{CC}$ types and $78 \mathrm{ST}$ of $S$. aureus isolates in this study. The three most predominant were CC5, CC7, and CC59. For MRSA isolates, there were $11 \mathrm{CC}$ types and $30 \mathrm{STs}$ in this study. The three most predominant were CC5, CC59, and CC45. The distributions of CCs on $S$. aureus $\left(\chi^{2}=0.033, P=0.678\right)$ and MRSA $\left(\chi^{2}=0.121, P=0.501\right)$ isolates between mothers and neonates had no significant differences (Figure 2).

In addition, zero, one, three, and zero pairs were positive for $P v l, T s t, E t a$, and $E t b$ genes, respectively. Four pairs were SCCmec type IV, one was type II, and one was NT. CC5 and ST188 were the most predominant CC and ST, respectively. Fourteen $(70.0 \%)$ pairs showed the same molecular characteristics including toxin genes, SCCmec type, CC, and ST (Figure 1).

\section{Discussion}

The prevalence of $S$. aureus nasal carriage in pregnant women was $25.8 \%$. This rate is similar to what has been observed in other studies. ${ }^{20,33}$ However, the prevalence of MRSA was $5.7 \%$, which is higher than that has been observed in other studies. ${ }^{13,33,34}$ The prevalence of $S$. aureus vaginal carriage in pregnant women was $7.3 \%$, which was higher than that in an American study $(2.3 \%, 10 / 432),{ }^{35}$ a Colombian study $(5.0 \%, 5 / 100),{ }^{36}$ and a French study (5.9\%, 67/1139). ${ }^{17}$ The prevalence of MRSA vaginal carriage in pregnant women was $1.7 \%$, which is higher than that in two American studies $\left([0.2 \%, 1 / 432]^{35}\right.$ and $[0.7 \%$, $\left.19 / 2702]^{12}\right)$, a Colombian study $(1.0 \%, 1 / 100),{ }^{36}$ a Chinese study $(0.5 \%, 15 / 2963),{ }^{9}$ and a Japanese study $(0 \%$, 0/4067). ${ }^{37}$ In the current study, the prevalence of $S$. aureus and MRSA vaginal carriage was lower than that of nasal carriage in pregnant women, which was similar to other observed studies. ${ }^{13,38}$ The prevalence of $S$. aureus with 


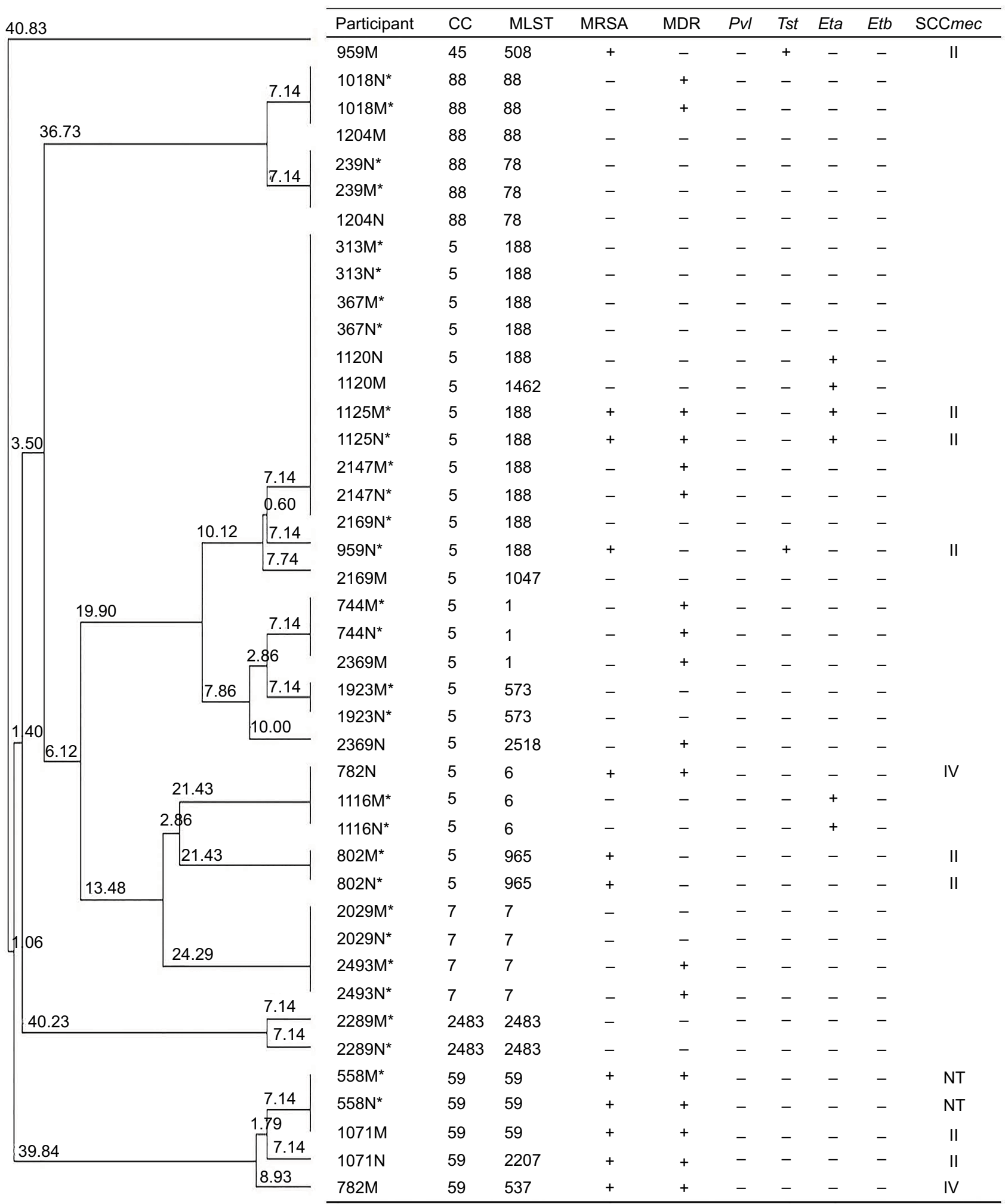

Figure I Clonal dendrogram and detailed information of Staphylococcus aureus for mother-neonate pairs in Shenzhen, 2015.

Notes: *This pair was concordant (with the same phenotypic and molecular characteristics). MDR, multidrug resistance, resistant to no less than three antibiotic classes. Abbreviations: M, mother; N, neonate; CC, clonal complex; MLST, multilocus sequence typing; MRSA, methicillin-resistant S. aureus; Pvl, Panton-Valentine leukocidin; Tst, toxic shock syndrome toxin; Eta, exfoliative toxin A; Etb, exfoliative toxin B; SCCmec, staphylococcal cassette chromosome mec; NT, non-typeable. 
Table 3 Phenotypic and toxin genetic characteristics of Staphylococcus aureus and MRSA isolates between neonates and mothers (nares and vagina) in Shenzhen, 2015, $\mathrm{n}(\%)$

\begin{tabular}{|c|c|c|c|c|c|c|c|c|c|c|}
\hline \multirow[t]{2}{*}{ Characteristics } & \multicolumn{5}{|l|}{ S. aureus } & \multicolumn{5}{|l|}{ MRSA } \\
\hline & $\begin{array}{l}\text { Neonates } \\
(n=60)\end{array}$ & $\begin{array}{l}\text { Mothers, } \\
\text { nasal } \\
(n=473)\end{array}$ & $\begin{array}{l}\text { Mothers, } \\
\text { vaginal } \\
(n=133)\end{array}$ & $P$-value ${ }^{a}$ & $P$-value ${ }^{b}$ & $\begin{array}{l}\text { Neonates } \\
(n=15)\end{array}$ & $\begin{array}{l}\text { Mothers, } \\
\text { nasal } \\
(n=104)\end{array}$ & $\begin{array}{l}\text { Mothers, } \\
\text { vaginal } \\
(n=3 I)\end{array}$ & $P$-value ${ }^{a}$ & $P$-value \\
\hline \multicolumn{11}{|c|}{ Phenotypic (resistant) } \\
\hline EM & $28(46.7)$ & $224(47.4)$ & $67(50.4)$ & 0.920 & 0.633 & $13(86.7)$ & $80(76.9)$ & $24(77.4)$ & 0.518 & 0.696 \\
\hline $\mathrm{PCN}$ & $56(93.3)$ & 433 (9I.5) & $123(92.5)$ & 0.635 & 1.000 & $13(86.7)$ & I0I (97.I) & $29(93.6)$ & 0.119 & 0.587 \\
\hline GM & $5(8.3)$ & $31(6.6)$ & II (8.3) & 0.585 & 1.000 & $2(13.3)$ & $6(5.8)$ & $5(16.1)$ & 0.265 & 1.000 \\
\hline $\mathrm{CM}$ & $17(28.3)$ & $163(34.5)$ & $55(4 \mid .4)$ & 0.344 & 0.083 & $10(66.7)$ & $60(57.7)$ & $20(64.5)$ & 0.509 & 0.886 \\
\hline RFP & $5(8.3)$ & $37(7.8)$ & $3(2.3)$ & 0.802 & 0.110 & $3(20.0)$ & $25(24.0)$ & $2(6.5)$ & 1.000 & 0.311 \\
\hline LZD & $2(3.3)$ & $16(3.4)$ & $5(3.8)$ & 1.000 & 1.000 & I (6.7) & $5(4.8)$ & $3(9.7)$ & 0.563 & 1.000 \\
\hline MXF & $3(5.0)$ & $40(8.5)$ & $18(13.5)$ & 0.457 & 0.078 & $3(20.0)$ & $6(5.8)$ & $9(29.0)$ & 0.086 & 0.723 \\
\hline TMP/SMX & $8(13.3)$ & $40(8.5)$ & $19(14.3)$ & 0.214 & 0.860 & $4(26.7)$ & $13(12.5)$ & $6(19.4)$ & 0.227 & 0.706 \\
\hline TEC & $12(20.0)$ & $227(48.0)$ & $108(8 \mid .2)$ & 0.000 & 0.000 & $4(26.7)$ & $54(51.9)$ & $23(74.2)$ & 0.067 & 0.002 \\
\hline TOB & II (I8.3) & $109(23.0)$ & $36(27.1)$ & 0.410 & 0.191 & $2(13.3)$ & $21(20.2)$ & $12(38.7)$ & 0.733 & 0.099 \\
\hline MDR & $23(38.3)$ & $244(51.6)$ & $86(64.7)$ & 0.053 & 0.001 & $12(80.0)$ & $83(79.8)$ & $26(83.9)$ & 1.000 & 1.000 \\
\hline \multicolumn{11}{|c|}{ Toxin genetic (positive) } \\
\hline Pvl & $3(5.0)$ & $22(4.7)$ & $3(2.3)$ & 0.753 & 0.377 & $0(0)$ & $13(12.5)$ & $2(6.5)$ & 0.368 & 1.000 \\
\hline Tst & $2(3.3)$ & II (2.3) & $3(2.3)$ & 0.648 & 0.647 & $0(0)$ & $3(2.9)$ & $\mathrm{I}(3.2)$ & 1.000 & 1.000 \\
\hline Eta & $7(11.7)$ & $13(2.8)$ & $4(3.0)$ & 0.004 & 0.037 & $2(13.3)$ & $2(1.9)$ & $\mathrm{I}(3.2)$ & 0.077 & 0.244 \\
\hline Etb & $0(0)$ & $\mathrm{I}(0.2)$ & $0(0)$ & 1.000 & NA & $0(0)$ & I (I.0) & $0(0)$ & 1.000 & NA \\
\hline
\end{tabular}

Notes: a $P$-value was calculated as the proportion of $S$. aureus/MRSA isolates between neonates and mothers' nares. ${ }^{\circ}$-value was calculated as the proportion of $S$. aureus/ MRSA isolates between neonates and mothers' vaginas. MDR, multidrug resistance, resistant to no less than three antibiotic classes.

Abbreviations: MRSA, methicillin-resistant S. aureus; n, number of isolates; EM, erythromycin; PCN, penicillin; GM, gentamicin; CM, clindamycin; RFP, rifampicin; LZD, linezolid; MXF, moxifloxacin; TEC, teicoplanin; TOB, tobramycin; TMP/SMX, trimethoprim-sulfamethoxazole; Pvl, Panton-Valentine leukocidin; Tst, toxic shock syndrome toxin; Eta, exfoliative toxin A; Etb, exfoliative toxin B; NA, not applicable.

both nasal and vaginal carriage among pregnant women was $3.3 \%$, which was higher than a Colombian study $(2.0 \%, 2 / 100) .{ }^{36}$ The prevalence of MRSA with both nasal and vaginal carriage among pregnant women was $0.5 \%$, which was higher than a Colombian study $(0 \%, 0 / 100) .{ }^{36}$ The prevalence results demonstrate the higher burden of MRSA than $S$. aureus isolates, which might result from the overuse of antibiotics during pregnancy in China and should be further explored. The higher burden of vaginal isolates than nasal isolates among pregnant women illustrates that pregnant women should pay greater attention to their vaginal cleanings.

The incidence of $S$. aureus carriage in neonates was $3.3 \%$, which was lower than that of healthy neonates within 6 days of birth in a Japanese study $(10.1 \%, 305 / 3020),{ }^{37}$ healthy neonates within 1 month of birth in an American study $(3.8 \%, 15 / 399),{ }^{35}$ neonates in a Greek study $(17.2 \%$, $22 / 128),{ }^{22}$ and neonates from the NICU in a Taiwanese study $(13.2 \%, 33 / 251) .{ }^{39}$ The incidence of MRSA carriage in neonates was $0.8 \%$, which was lower than that of neonates from an NICU in a Taiwanese study $(4.4 \%$, $11 / 251)^{39}$ and neonates from an NICU in two Italian studies $\left([25.9 \%, 187 / 722]^{40}\right.$ and $\left.[22.9 \%, 217 / 949]^{3}\right)$ but higher than healthy neonates within 6 days of birth in a Japanese study $(0.4 \%, 12 / 3020)^{37}$ and healthy neonates within 1 month of birth in an American study $(0.3 \%, 1 / 399) .{ }^{35}$ The results demonstrated that the incidence of $S$. aureus carriage in neonates in the current study was lower than that in many other countries and regions. This might be related to the good hygiene of mothers and clean hospital environments. However, the incidence of MRSA carriage in neonates was lower than that in NICU neonates but was higher than that in healthy neonates from other countries and regions, which demonstrates that the incidence of MRSA carriage in neonates of this current study was not low and should be paid greater attention. Moreover, the prevalence of isolate carriage among 414 mothers with caesarian section was as follows: S. aureus (nasal, 28.0\%; vaginal, 8.5\%; and nasal and vaginal: $3.6 \%$ ) and MRSA (nasal, 5.3\%; vaginal, 2.2\%; and nasal and vaginal: $1.0 \%$ ). We found that several neonates not born by vaginal delivery had $S$. aureus $(1.5 \%$, $6 / 414)$ and/or MRSA $(0.5 \%, 2 / 414)$ carriage, which might be transmitted from $S$. aureus and MRSA contamination in the hospital environment or $S$. aureus and MRSA carriage on the hands of health care workers; this possibility requires further exploration. 

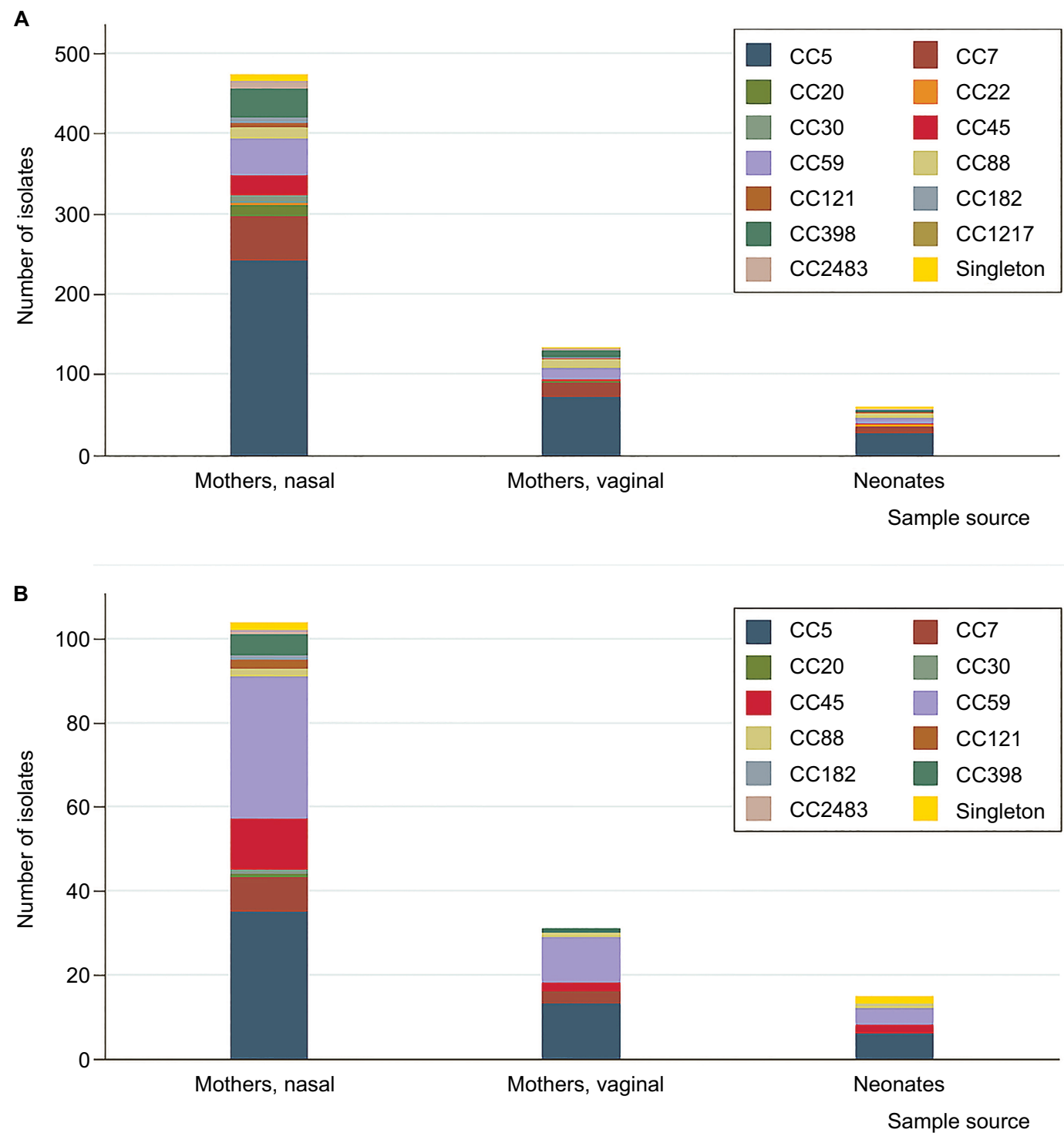

Figure 2 Distribution of CCs by neonates and mothers.

Notes: Singleton refers to STs that could not be assigned to any group in Shenzhen, 2015. (A) Distribution of CCs on Staphylococcus aureus isolates by neonates and mothers. (B) Distribution of CCs on MRSA isolates by neonates and mothers.

Abbreviations: CC, clonal complex; ST, sequence type; MRSA, methicillin-resistant S. aureus.

In the current study, neonates born through vaginal delivery were 2.9 times more likely to have $S$. aureus carriage, which was similar to other studies..$^{21,41}$ Male neonates were 1.7 times more likely to have $S$. aureus carriage than female neonates, which was similar to other studies. ${ }^{42,43}$ Neonates whose mothers had a low frequency of vaginal examination after hospitalization $(\leq 2)$ were 1.8 times more likely to have $S$. aureus carriage, which might be explained by the fact that mothers with a high frequency of vaginal examination might have an increased awareness of the importance of vaginal cleaning; this should be further explored. Low frequency of vaginal examination after hospitalization $(\leq 2)$ was a risk factor for MRSA carriage among neonates.

The proportions of antibiotic resistance in $S$. aureus and MRSA isolates among both mothers and neonates were similar to other studies. ${ }^{4,6,17,22}$ However, the proportions of MDR in neonatal MRSA isolates, maternal nasal MRSA isolates, and maternal vaginal MRSA isolates were $80.0 \%, 79.8 \%$, and $83.9 \%$, respectively, which were higher than other published studies and should be noticed by health care workers when selecting antibiotics. ${ }^{6,17,22,44}$ There were few significantly different proportions of antibiotic resistance of $S$. aureus and MRSA isolates between 
the mothers and neonates, which might demonstrate the similar antibiotic-resistant spectrum of isolates between mothers and neonates.

The proportions of toxin genes in S. aureus and MRSA isolates among both mothers and neonates were also similar to other studies. ${ }^{3,20,22,36,44}$ Moreover, there were few significantly different proportions of toxin genes of $S$. aureus and MRSA isolates between the mothers and neonates, which might demonstrate the similar toxin rates of isolates between mothers and neonates.

Most MRSA nasal isolates among the mothers and neonates were CA-MRSA, but most MRSA vaginal isolates of the mothers were HA-MRSA. This result was similar to other studies and might demonstrate the presence of crosstransmission between communities and hospitals. ${ }^{3,7,21,40}$

CC5, the most predominant CC type of $S$. aureus and MRSA isolates, was reported to be the main CC type of HA-MRSA. ${ }^{45}$ The distributions of CC types of $S$. aureus and MRSA isolates between the mothers and neonates were similar and demonstrated that there was a phylogenetic relatedness of isolates between the mothers and neonates.

We found that there were positive associations of $S$. aureus carriage between the mothers and neonates. The aRR and $95 \%$ CI between $S$. aureus carriage of the neonates and two-site carriage (both $S$. aureus nasal and vaginal carriage) of the mothers were higher than single $S$. aureus carriage, which suggests the higher risk of the two-site carriage. The aRR of $S$. aureus nasal carriage of the mothers among neonates was higher than that in an African study (odds ratio [OR], 2.5; 95\% CI, 1.6-4.0). ${ }^{19}$ The aRR of $S$. aureus vaginal carriage of the mothers among neonates was higher than that in an American study (RR, 4.8; 95\% CI, 2.4-9.5), ${ }^{21}$ an Israelite study (RR, 5.7; 95\% CI, 2.3-13.9), ${ }^{18}$ a Dutch study (OR, 2.0; 95\% CI, 1.2-3.6), ${ }^{46}$ and an African study (OR, 2.6; 95\% CI, 1.3-4.9). ${ }^{19}$ The results demonstrated that $S$. aureus vertical transmission is more hazardous on $S$. aureus carriage in neonates in China. In addition, we performed analyses for NNHs regarding the carriage of $S$. aureus isolates and showed that the potential harms were similar to the results of the RRs. Therefore, our findings suggest that $S$. aureus maternal carriage plays a role in the $S$. aureus carriage of neonates. Further research is necessary to determine whether effective decolonization of $S$. aureus carriage in mothers is able to reduce the risk of $S$. aureus carriage in neonates.

This study contributes to the existing literature because we assessed the maternal-neonatal associations of $S$. aureus and MRSA carriage in China, which has not been previously published. The phenotypic characteristics of $S$. aureus and MRSA isolates between mothers and neonates were significantly different, indicating that the phenotypic characteristics of the isolates between mothers and neonates are similar. With regard to the molecular characteristics of $S$. aureus and MRSA isolates between mothers and neonates, the differences of most genes were insignificant, which could also illustrate that the molecular characteristics of isolates between mothers and neonates are similar. Moreover, 14 maternal-neonatal pairs showed the same culture results, phenotypic and molecular characteristics. From these aspects, we could assess that there was transmission of $S$. aureus and MRSA between the mothers and neonates.

The strengths of this study are that it used a longitudinal cohort design in which the participants and researchers were blinded to the exposure status to answer a directed research question about whether $S$. aureus or MRSA carriage of mothers is an independent predictor of $S$. aureus or MRSA carriage of neonates. We accounted for other potential influencing factors for $S$. aureus and MRSA carriage of neonates in our analyses. Second, we sampled multiple body sites to account for their potential contribution. Cultures from one body site cannot identify all $S$. aureus and MRSA carriers, and cultures from multiple sites (versus the nares only) may increase the sensitivity of screening for $S$. aureus and MRSA. ${ }^{38}$ Third, the sample size of this study was relatively larger than many other published studies. ${ }^{17,18,20-22}$ Finally, to our knowledge, this study helped elucidate the maternal-neonatal association of $S$. aureus and MRSA isolates not only by culture results but also through the phenotypic and molecular characteristics of isolates.

This study also has some limitations. First, environmental contamination of $S$. aureus and MRSA of hospitals and $S$. aureus and MRSA carriage of health care workers could not be taken into consideration because of limited funding. Second, we could not explore the persistent status of $S$. aureus and MRSA carriage in both mothers and neonates because of limited time. Third, we do not offer decolonization to neonates and whether this intervention would improve the health of neonates is scientifically unproven. Fourth, there may be some bias in reaching the conclusion of the study results in regard to high, moderate, or low carriage rates because of the different study designs, swab acquisition age, and swab site of the cited studies. Finally, whole-genome sequencing would further strengthen this study. 


\section{Conclusion}

Notwithstanding these limitations, our findings suggest that $S$. aureus carriage of mothers increases the risk for $S$. aureus carriage among neonates. The decolonization of mothers with S. aureus, including strengthening disinfection measures and education of hand hygiene, should be considered as strategies to prevent $S$. aureus carriage among neonates.

\section{Acknowledgments}

This study was supported by the Innovation Fund of Guangdong Science and Technology Planning Project (grant number 2014A020213013). The funders have no role in the study design, data collection and analysis, decision to publish, or preparation of the manuscript.

\section{Author contributions}

Jialing Lin and Zhenjiang Yao designed the study, performed experiments, analyzed data, and wrote the manuscript; Chuanan $\mathrm{Wu}$ and Chunrong Yan collected samples and performed experiments; Qianting Ou and Dongxin Lin collected information and supervised experiments; and Junli Zhou and Xiaohua Ye analyzed data. All authors contributed toward data analysis, drafting and revising the paper and agree to be accountable for all aspects of the work.

\section{Disclosure}

The authors report no conflicts of interest in this work.

\section{References}

1. Reich PJ, Boyle MG, Hogan PG, et al. Emergence of communityassociated methicillin-resistant Staphylococcus aureus strains in the neonatal intensive care unit: an infection prevention and patient safety challenge. Clin Microbiol Infect. 2016;22(7):645.e641-645.e648.

2. Kriebs JM. Staphylococcus infections in pregnancy: maternal and neonatal risks. J Perinat Neonatal Nurs. 2016;30(2):115-123.

3. Giuffre M, Amodio E, Bonura C, et al. Methicillin-resistant Staphylococcus aureus nasal colonization in a level III neonatal intensive care unit: incidence and risk factors. Am J Infect Control. 2015;43(5):476-481.

4. Mumtaz S, Ahmad M, Aftab I, Akhtar N, ul Hassan M, Hamid A. Aerobic vaginal pathogens and their sensitivity pattern. JAyub Med Coll Abbottabad. 2008;20(1):113-117.

5. Sobel JD. Vulvovaginal candidosis. Lancet. 2007;369(9577):1961-1971.

6. Abdelaziz ZA, Ibrahim ME, Bilal NE, Hamid ME. Vaginal infections among pregnant women at Omdurman Maternity Hospital in Khartoum, Sudan. J Infect Dev Ctries. 2014;8(4):490-497.

7. Creech CB, Litzner B, Talbot TR, Schaffner W. Frequency of detection of methicillin-resistant Staphylococcus aureus from rectovaginal swabs in pregnant women. Am J Infect Control. 2010;38(1):72-74.

8. Andrews WW, Schelonka R, Waites K, Stamm A, Cliver SP, Moser S. Genital tract methicillin-resistant Staphylococcus aureus: risk of vertical transmission in pregnant women. Obstet Gynecol. 2008;111(1):113-118.

9. Chen KT, Huard RC, Della-Latta P, Saiman L. Prevalence of methicillinsensitive and methicillin-resistant Staphylococcus aureus in pregnant women. Obstet Gynecol. 2006;108(3 Pt 1):482-487.
10. Chen KT, Campbell H, Borrell LN, Huard RC, Saiman L, Della-Latta P. Predictors and outcomes for pregnant women with vaginal-rectal carriage of community-associated methicillin-resistant Staphylococcus aureus. Am J Perinatol. 2007;24(4):235-240.

11. Beigi R, Hanrahan J. Staphylococcus aureus and MRSA colonization rates among gravidas admitted to labor and delivery: a pilot study. Infect Dis Obstet Gynecol. 2007;2007:70876.

12. Top KA, Buet A, Whittier S, Ratner AJ, Saiman L. Predictors of Staphylococcus aureus rectovaginal colonization in pregnant women and risk for maternal and neonatal infections. $J$ Pediatric Infect Dis Soc. 2012;1(1):7-15.

13. Reusch M, Ghosh P, Ham C, Klotchko A, Singapuri S, Everett G. Prevalence of MRSA colonization in peripartum mothers and their newborn infants. Scand J Infect Dis. 2008;40(8):667-671.

14. Zervou FN, Zacharioudakis IM, Ziakas PD, Mylonakis E. MRSA colonization and risk of infection in the neonatal and pediatric ICU: a meta-analysis. Pediatrics. 2014;133(4):e1015-e1023.

15. Li S, Ning X, Song W, et al. Clinical and molecular characteristics of community-acquired methicillin-resistant Staphylococcus aureus infections in Chinese neonates. APMIS. 2015;123(1):28-36.

16. Carey AJ, Duchon J, Della-Latta P, Saiman L. The epidemiology of methicillin-susceptible and methicillin-resistant Staphylococcus aureus in a neonatal intensive care unit, 2000-2007. J Perinatol. 2010;30(2):135-139.

17. Bourgeois-Nicolaos N, Lucet JC, Daubie C, et al. Maternal vaginal colonisation by Staphylococcus aureus and newborn acquisition at delivery. Paediatr Perinat Epidemiol. 2010;24(5):488-491.

18. Leshem E, Maayan-Metzger A, Rahav G, et al. Transmission of Staphylococcus aureus from mothers to newborns. Pediatr Infect Dis J. 2012;31(4):360-363.

19. Roca A, Bojang A, Camara B, et al. Maternal colonization with Staphylococcus aureus and Group B streptococcus is associated with colonization in newborns. Clin Microbiol Infect. 2017;23(12): 974-979.

20. Schaumburg F, Alabi AS, Mombo-Ngoma G, et al. Transmission of Staphylococcus aureus between mothers and infants in an African setting. Clin Microbiol Infect. 2014;20(6):O390-O396.

21. Jimenez-Truque N, Tedeschi S, Saye EJ, et al. Relationship between maternal and neonatal Staphylococcus aureus colonization. Pediatrics. 2012;129(5):e1252-e1259.

22. Chatzakis E, Scoulica E, Papageorgiou N, Maraki S, Samonis G, Galanakis E. Infant colonization by Staphylococcus aureus: role of maternal carriage. Eur J Clin Microbiol Infect Dis. 2011;30(9):1111-1117.

23. Pinter DM, Mandel J, Hulten KG, Minkoff H, Tosi MF. Maternalinfant perinatal transmission of methicillin-resistant and methicillinsensitive Staphylococcus aureus. Am J Perinatol. 2009;26(2): 145-151.

24. Brigtsen AK, Jacobsen AF, Dedi L, Melby KK, Fugelseth D, Whitelaw A. Maternal colonization with group B Streptococcus is associated with an increased rate of infants transferred to the neonatal intensive care unit. Neonatology. 2015;108(3):157-163.

25. Cassidy-Bushrow AE, Sitarik A, Levin AM, et al. Maternal group B Streptococcus and the infant gut microbiota. $J$ Dev Orig Health Dis. 2016;7(1):45-53.

26. Magiorakos AP, Srinivasan A, Carey RB, et al. Multidrug-resistant, extensively drug-resistant and pandrug-resistant bacteria: an international expert proposal for interim standard definitions for acquired resistance. Clin Microbiol Infect. 2012;18(3):268-281.

27. Su LT, Liang JL, Guo XJ, Feng F. Comparison of methods for DNA extraction in Staphylococcus aureus. J Zhongkai Univ Agric Eng. 2011; 24(1):15-19.

28. McClure JA, Conly JM, Lau V, et al. Novel multiplex PCR assay for detection of the staphylococcal virulence marker Panton-Valentine leukocidin genes and simultaneous discrimination of methicillin-susceptible from -resistant staphylococci. J Clin Microbiol. 2006;44(3):1141-1144. 
29. Jarraud S. Relationships between Staphylococcus aureus genetic background, virulence factors, agr groups (alleles), and human disease. Infect Immun. 2002;70(2):631-641.

30. Zhang K, McClure JA, Elsayed S, Louie T, Conly JM. Novel multiplex PCR assay for characterization and concomitant subtyping of staphylococcal cassette chromosome mec types I to $\mathrm{V}$ in methicillin-resistant Staphylococcus aureus. J Clin Microbiol. 2005;43(10):5026-5033.

31. Enright MC, Day NP, Davies CE, Peacock SJ, Spratt BG. Multilocus sequence typing for characterization of methicillin-resistant and methicillin-susceptible clones of Staphylococcus aureus. J Clin Microbiol. 2000;38(3):1008-1015.

32. Altman DG, Andersen PK. Calculating the number needed to treat for trials where the outcome is time to an event. BMJ. 1999;319(7223):1492-1495.

33. Garcia CP, Rosa JF, Cursino MA, et al. Non-multidrug-resistant, methicillin-resistant Staphylococcus aureus in a neonatal unit. Pediatr Infect Dis J. 2014;33(10):e252-e259.

34. Patel RI, Kaufman HK. Nasopharyngeal carriage of methicillin-resistant Staphylococcus aureus: incidence and outcomes in pregnant women. J Am Osteopath Assoc. 2011;111(6):389-395.

35. James L, Gorwitz RJ, Jones RC, et al. Methicillin-resistant Staphylococcus aureus infections among healthy full-term newborns. Arch Dis Child Fetal Neonatal Ed. 2008;93(1):F40-F44.

36. Correa O, Delgado K, Rangel C, Bello A, Reyes N. Nasal and vaginal colonization of methicillin-resistant Staphylococcus aureus in pregnant women in Cartagena, Colombia. Colombia Medica. 2012;43(1):19-27.

37. Mitsuda T, Arai K, Fujita S, Yokota S. Demonstration of mother-to-infant transmission of Staphylococcus aureus by pulsed-field gel electrophoresis. Eur J Pediatr. 1996;155(3):194-199.
38. Andrews JI, Fleener DK, Messer SA, Kroeger JS, Diekema DJ. Screening for Staphylococcus aureus carriage in pregnancy: usefulness of novel sampling and culture strategies. Am J Obstet Gynecol. 2009;201(4):396. e391-396.e395.

39. Kuo CY, Huang YC, Huang DTN, et al. Prevalence and molecular characterization of Staphylococcus aureus colonization among neonatal intensive care units in Taiwan. Neonatology. 2014;105(2):142-148.

40. Geraci DM, Giuffre M, Bonura C, et al. Methicillin-resistant Staphylococcus aureus colonization: a three-year prospective study in a neonatal intensive care unit in Italy. PLoS One. 2014;9(2):e87760.

41. Huang YC, Chao AS, Chang SD, et al. Association of Staphylococcus aureus colonization in parturient mothers and their babies. Pediatr Infect Dis J. 2009;28(8):742-744.

42. Donovan JT, Horace MG, Theodore FH, Russell RR, Kenneth DR. Sex distribution of Staphylococcus aureus colonization and disease in newborn infants. N Engl J Med. 1963;269:337-341.

43. Halablab MA, Hijazi SM, Fawzi MA, Araj GF. Staphylococcus aureus nasal carriage rate and associated risk factors in individuals in the community. Epidemiol Infect. 2010;138(5):702-706.

44. Lamaro-Cardoso J, de Lencastre H, Kipnis A, et al. Molecular epidemiology and risk factors for nasal carriage of staphylococcus aureus and methicillin-resistant $\mathrm{S}$. aureus in infants attending day care centers in Brazil. J Clin Microbiol. 2009;47(12):3991-3997.

45. Otto M. MRSA virulence and spread. Cell Microbiol. 2012;14(10): 1513-1521.

46. Lebon A, Moll HA, Tavakol M, et al. Correlation of bacterial colonization status between mother and child: the generation R study. J Clin Microbiol. 2010;48(3):960-962.
Infection and Drug Resistance

\section{Publish your work in this journal}

Infection and Drug Resistance is an international, peer-reviewed openaccess journal that focuses on the optimal treatment of infection (bacterial, fungal and viral) and the development and institution of preventive strategies to minimize the development and spread of resistance. The journal is specifically concerned with the epidemiology of antibiotic

\section{Dovepress}

resistance and the mechanisms of resistance development and diffusion in both hospitals and the community. The manuscript management system is completely online and includes a very quick and fair peerreview system, which is all easy to use. Visit http://www.dovepress.com/ testimonials.php to read real quotes from published authors. 